\title{
Review
}

Verena Kanoldta ${ }^{a}$, Lisa Fischer ${ }^{\mathrm{a}}$ and Carsten Grashoff*

\section{Unforgettable force - crosstalk and memory of mechanosensitive structures}

https://doi.org/10.1515/hsz-2018-0328

Received July 17, 2018; accepted November 11, 2018; previously published online November 22, 2018

\begin{abstract}
The ability of cells to sense and respond to mechanical stimuli is crucial for many developmental and homeostatic processes, while mechanical dysfunction of cells has been associated with numerous pathologies including muscular dystrophies, cardiovascular defects and epithelial disorders. Yet, how cells detect and process mechanical information is still largely unclear. In this review, we outline major mechanisms underlying cellular mechanotransduction and we summarize the current understanding of how cells integrate information from distinct mechanosensitive structures to mediate complex mechanoresponses. We also discuss the concept of mechanical memory and describe how cells store information on previous mechanical events for different periods of time.
\end{abstract}

Keywords: biophysics; cell biology; mechanical memory; mechanotransduction.

\section{Introduction}

Cells are exposed to a complex mechanical environment. Endothelial cells in our blood vessel, for instance, experience shear forces of the blood flow and blood pressure. Muscle cells feel various degrees of contractile forces, chondrocytes are exposed to compressive loads, and keratinocytes have to adapt to external stimuli caused by

aerena Kanoldt and Lisa Fischer: These authors contributed equally.

*Corresponding author: Carsten Grashoff, Group of Molecular Mechanotransduction, Max Planck Institute of Biochemistry, 82152 Martinsried, Germany; and Department of Quantitative Cell Biology, Institute of Molecular Cell Biology, University of Münster, 48149 Münster, Germany, e-mail: grashoff@uni-muenster.de. https://orcid.org/0000-0003-0118-9231

Verena Kanoldt and Lisa Fischer: Group of Molecular Mechanotransduction, Max Planck Institute of Biochemistry, 82152 Martinsried, Germany compression, shear and strain of our skin. In addition, cells generate their own mechanical forces and pull on one another or on extracellular networks, for example during tissue formation or collective cell migration. We know that cells adapt to those kinds of mechanical stimulations and efficiently sense magnitude, direction and frequency of the applied forces (Hoffman et al., 2011). It is also clear that cells' ability to translate mechanical stimuli into biochemical information plays an important role for numerous pathophysiological processes (Jaalouk and Lammerding, 2009), but we are just beginning to develop a molecular understanding of mechanotransduction in cells. We will therefore highlight important subcellular structures that govern the sensation and propagation of mechanical stimuli, and describe how cells integrate signals from those distinct mechanosensitive elements. We then discuss how cells store mechanical information to develop a mechanical memory.

\section{Cells are inherently mechanosensitive objects}

A prevailing misconception is that cellular mechanotransduction is orchestrated by just a few mechanosensitive proteins - often called 'mechanosensors' - that singlehandedly mediate a distinct force response. Certainly, there are proteins that fulfill very specific biomechanical functions, but it is important to recognize that virtually the whole cell or at least many of its subcellular structures are inherently mechanosensitive (Figure 1). The plasma membrane and the underlying cortical actin network, for instance, are sensitive to mechanical stimulations at the cell surface and quickly remodel in response to changes in plasma membrane tension, shear or bending (Gauthier et al., 2012; Mueller et al., 2017). The three major cytoskeletal networks - actin filaments, intermediate filaments and microtubules - exhibit specific responses to mechanical stimuli (Gardel et al., 2008) as well as their connected cell adhesion structures such as focal adhesions (FAs), adherens junctions (AJs) and desmosomes (Geiger et al., 
2009; Rubsam et al., 2017). Trafficking at the Golgi apparatus is modulated by mechanically constraining cells (Guet et al., 2014), while mitochondrial activity is boosted by the application of externally applied stretch (BartolakSuki et al., 2015). The composition of the nuclear membrane is sensitive to tissue stiffness (Swift et al., 2013), and kinetochores as well as their associated microtubules integrate mechanical signals at the mitotic spindle during cell division (Maresca and Salmon, 2010; Forth and Kapoor, 2017).

If cells are endowed with so many mechanosensitive entities, then complex mechanical inputs must be

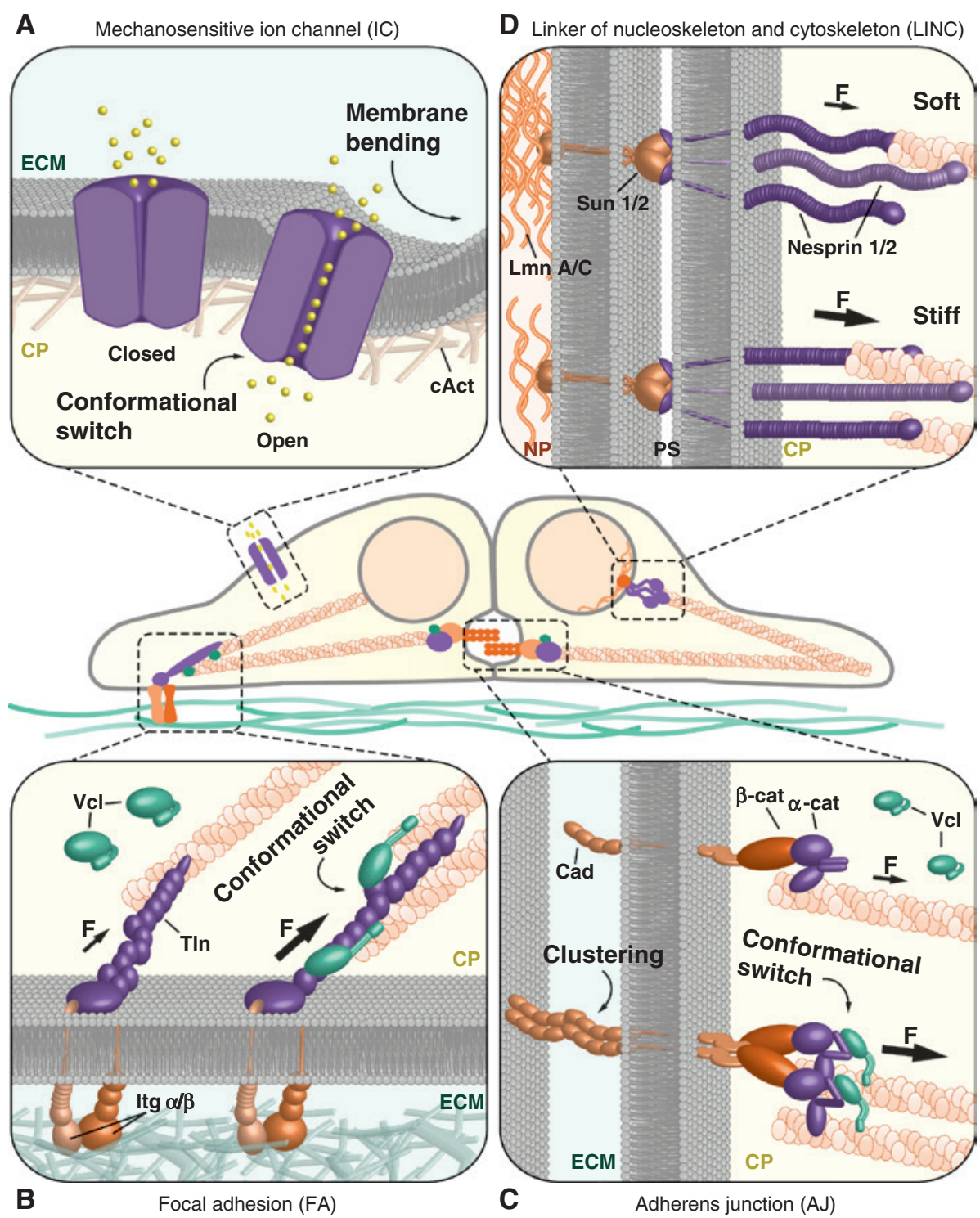

Figure 1: Schematic overview of representative mechanosensitive structures.

(A) Mechanosensitive (MS) ion channels perceive changes in plasma membrane tension, which can be modulated by the cortical actin network (cAct). Mechanical forces are thought to gate MS ion channels by inducing a conformational switch resulting in pore opening and ion flux; cytoplasm (CP), extracellular matrix (ECM). (B) Focal adhesions (FAs) sense and transmit mechanical forces during cell-adhesion to the ECM. FAs contain hundreds of proteins including talin (TIn), which connects the ECM-binding integrin receptors (Itg) to the actin cytoskeleton. In response to increased tension, Tln reveals cryptic binding sites that lead to the recruitment of Vinculin (Vcl). (C) Adherens junctions (Al) mediate mechanotransduction at intercellular adhesion sites. They are composed of cadherin receptors (Cad), which connect to the actin cytoskeleton via $\alpha$ - and $\beta$-catenin ( $\alpha$-/ $\beta$-cat). Similar to FAs, mechanical forces lead to Vcl recruitment in Als through the exposure of a cryptic binding site in $\alpha$-cat. (D) The nuclear membrane perceives mechanical information through the 'linker of nucleoskeleton and cytoskeleton' (LINC) complex comprising Nesprins and Sun proteins that link the cytoskeletal network to the nucleoskeleton. Changes in substrate rigidity are transduced across the LINC complex and lead, for instance, to the modulation of lamin A/C (LmnA/C) expression levels; nucleoplasm (NP), perinuclear space (PS). 
processed by integrating signals from not only one but many mechanosensitive structures. Yet, our current understanding of how mechanochemical signaling networks synergize in cells is still limited and it remains challenging to develop a comprehensive picture of cellular mechanotransduction. To assist this process, we will highlight central mechanosensitive structures playing a prominent role in mechanotransduction and we discuss how signals from spatially distinct elements are integrated to mediate specific mechanoresponses. Finally, we will elaborate on the concept of mechanical memory that allows cells to remember their mechanical history.

\section{Mechanical signaling by mechanosensitive ion channels}

Most if not all cell types express ion channels that can be regulated by mechanical signals (Figure 1A, Ranade et al., 2015). The first examples of such mechanosensitive (MS) ion channels are MscL and MscS, which were discovered in Escherichia coli where they control the passage of ions and water in response to changes in osmotic pressure (Martinac et al., 1990; Perozo et al., 2002). Not long after, the potassium channels TREK and TRAAK were identified as critical mechanosensors in neurons (Maingret et al., 1999a,b), and members of the DEG/ENaC class of sodium channels were found to be important for touch sensation in Caenorhabditis elegans (Goodman et al., 2002; Chalfie, 2009). A famous example of a MS ion channel in higher animals is a macromolecular complex that resides in epithelial hair cells of the inner ear and translates sound waves into electric information (Siemens et al., 2004; Kazmierczak et al., 2007). Moreover, Piezo channels have received considerable attention. They present an evolutionary conserved family of MS ion channels (Coste et al., 2010) that play a key role in many mechanobiological processes including vascular development (Ranade et al., 2014a), touch sensation, proprioception and breathing (Ranade et al., 2014b; Woo et al., 2015; Nonomura et al., 2017).

Identifying the molecular details of how MS channels are gated by mechanical signals remains a challenging task. One reason is that these large transmembrane proteins are difficult to reconstitute in vitro. In addition, many MS ion channels are not exclusively sensitive to mechanical signals but also respond to non-mechanical stimuli like changes in $\mathrm{pH}$, temperature or voltage (Honore, 2007; Moroni et al., 2018). It nevertheless became obvious that certain MS ion channels sense mechanical signals in a highly specific fashion. Many of them, such as MscL, MscS, TREK and TRAAK, primarily respond to tension differentials across the lipid bilayer (Perozo et al., 2002; Honore, 2007; Martinac, 2011), which is consistent with the observation that the composition and stiffness of the lipid bilayer modulates MS ion activity (Chubinskiy-Nadezhdin et al., 2011; Qi et al., 2015). Other MS channels require the direct or indirect engagement with extracellular ligands and/or the cytoskeleton to be fully functional (Mazzochi et al., 2006; Cueva et al., 2007; Sharif-Naeini et al., 2009): the TRPP channel engages the actin crosslinker filamin A for gating (Sharif-Naeini et al., 2009), and the DEG/ENaC channel complex as well as NOMPC from the TRP family interact with microtubules (Goodman et al., 2002; Zhang et al., 2015).

As diverse as the force sensing mechanisms are the induced downstream cascades translating the mechanical into a biochemical signal. These events strongly depend on the mechanical input, the cell type and the respective ion selectivity of the expressed channels. The potassium channels TREKs and TRAAK, for instance, adjust the potential for hyperpolarization in neurons and thereby counteract the activity of non-selective Piezo channels (Brohawn et al., 2014a,2014b; Brohawn, 2015). ENaC calcium channels can initiate neurotransmitter release at the synapse (Hill et al., 2017), while Piezo-mediated calcium influxes can induce very specific cell type-dependent responses, such as stimulating transglutaminase activity in myocytes to induce the constriction of small blood vessels (Retailleau et al., 2015). As acute changes in ion concentrations, especially $\mathrm{Ca}^{2+}$ levels, affect a whole range of intracellular signaling cascades, MS ion channels impact cellular behavior in the short-term (Doyle et al., 2004), but can also lead to long-lasting changes in gene transcription activities (Berridge et al., 2003). In a nutshell, MS ion channels are highly versatile force transducers at the plasma membrane that modulate cellular processes in a context- and cell-type dependent fashion.

\section{Mechanotransduction at cell adhesion sites}

Mechanical signaling through cell adhesion receptors and their associated complexes endow cells with an additional mode of force transmission at the plasma membrane. A prominent example is the sensation of extracellular cues by FAs, which mediate the connection between the extracellular matrix (ECM) and the intracellular cytoskeleton (Figure 1B, Humphrey et al., 2014; Ringer et al., 2017a). FAs provide molecular specificity to the mechanotransduction process as their core constituents, the integrin receptors, engage distinct ECM components such as collagens, laminins or fibronectin. 
Integrins are heterodimeric transmembrane proteins and connect, with the exception of the keratin-engaging hemidesmosomal $\alpha 6 \beta 4$ integrin, to the actin cytoskeleton. The integrin-actin interaction, however, is not direct but mediated by a multitude of intracellular adapter molecules that bestow the FA with mechanosensitive properties (Geiger et al., 2009; Ringer et al., 2017b). Even though many of the underlying mechanisms are still incompletely understood, it appears that those FA proteins directly exposed to mechanical forces are also involved in processing the mechanical information. The most prominent example is the ubiquitously expressed FA protein talin, which binds and thereby activates integrin receptors and at the same time engages the actin cytoskeleton (Roberts and Critchley, 2009). Talin harbors numerous binding sites for another FA protein called vinculin, but those vinculin binding sites remain cryptic until mechanical tension across the integrin-talin-actin linkage induces conformational changes that facilitate vinculin binding (del Rio et al., 2009; Austen et al., 2015; Yao et al., 2016). The vinculin binding to actin filaments is stabilized by mechanical forces in an orientation dependent fashion (Huang et al., 2017) resulting in a force- and direction-dependent FA reinforcement, which is observed when cells are exposed to high matrix stiffness or externally applied mechanical loads (Pelham and Wang, 1997; Riveline et al., 2001; Pasapera et al., 2010).

A similar strengthening mechanism has been observed in other cell adhesion structures such as AJs, which mediate intercellular cohesion through the cadherin-catenin linkage (Figure 1C; Han and de Rooij, 2016). These complexes also connect to actin filaments and are stabilized in response to mechanical stretch (Buckley et al., 2014). As in FAs, vinculin is recruited to AJs in a force-sensitive fashion because the AJ-binding partner $\alpha$-catenin harbors a cryptic vinculin binding motif that becomes accessible under force (Yonemura et al., 2010; Yao et al., 2014a). Another well-known example of mechanical allosterism in adhesion complexes is the regulation of the cytoplasmic adaptor molecule p130Cas that becomes phosphorylated by Src family kinases upon exposure to mechanical stretch (Sawada et al., 2006).

As FAs and AJs comprise many proteins, in case of FAs hundreds (Kuo et al., 2011; Schiller et al., 2011), it is plausible that additional mechanisms of force-dependent regulation of protein activity occur in these structures. How local changes in distinct adhesion proteins lead to the modulation of chemical signaling cascades or transcriptional activities remains to be elucidated. It is certainly possible that mechanical force directly modulates the enzymatic activity of central kinases as has been suggested for focal adhesion kinase (FAK) and myosin light chain kinase (Zhou et al., 2015; Baumann et al., 2017). It has also become clear that Rho-GTPases are crucial for initiating an adhesion-dependent force response (Rottner et al., 1999) and there is strong evidence that transcriptional regulators like YAP/TAZ in FAs (Oria et al., 2017) and $\beta$-catenin in AJs (Gayrard et al., 2018) play an important role in mediating mechanoresponses. Still, the current understanding of mechano-chemical coupling in cell adhesion structures is fragmentary and will remain a matter of active research in the future.

\section{Mechanotransduction at the nucleus}

A very efficient way of translating mechanical signals into changes in gene expression is the so-called nuclear mechanotransduction (Figure 1D). Here, the underlying concept is that mechanical forces essentially bypass cytoplasmic signaling cascades to directly modulate the physical organization of the nuclear membrane or even the chromatin itself thereby modulating gene expression (Kirby and Lammerding, 2018). In this mode of cellular mechanotransduction, forces are transmitted across cytoskeletal networks that physically engage with the nuclear membrane through the "linker of nucleoskeleton and cytoskeleton' (LINC) complex comprising Nesprins at the outer and the Sun proteins (Sun1 and Sun2) at the inner nuclear membrane (Lombardi et al., 2011). Sun1 and Sun 2 connect to the nucleoskeleton by binding lamin $\mathrm{A} / \mathrm{C}$, two intermediate filament proteins that are essential for nuclear integrity and regulated by mechanical stress (Swift et al., 2013). As mutations in the LINC complex as well as lamin $\mathrm{A} / \mathrm{C}$ are associated with a wide range of pathologies including severe muscular dystrophies (Worman, 2012; Stroud et al., 2014), it is beyond question that nuclear mechanotransduction is crucial for mechanical homeostasis of cells, especially when they are exposed to significant external loads.

How the mechanical signals at the nuclear membrane are translated into a distinct response within the nucleus is still debated. Potential mechanisms include the force-dependent regulation of cryptic lamin phosphorylation sites through mechanical allosterism (Swift et al., 2013), effects on chromatin condensation (Tajik et al., 2016), changes in the architecture of Cajal bodies (Poh et al., 2012), and mechanical regulation of nuclear pore permeability (Jahed et al., 2016; Elosegui-Artola et al., 2017). Clearly, the nucleus is yet another highly mechanosensitive organelle and crucial for the mechanobiology of cells. 


\section{Mechanotransduction at the cytoskeleton}

Our examples seem to indicate that the actin cytoskeleton plays an important role for cellular mechanotransduction simply by connecting mechanosensitive elements, for example, cell surface receptors with intracellular networks or the nucleus. It is important to realize, however, that actin networks actively sense mechanical signals and do so in at least two different ways (Galkin et al., 2012; Harris et al., 2018). First, mechanical forces modulate the binding affinity of actin interactors: The recruitment of the actin severing protein cofilin, for instance, is impaired when mechanical tension acts across the actin filament (Hayakawa et al., 2011), whereas binding of myosin motors to actin increases under force (Laakso et al., 2008; Uyeda et al., 2011). Second, mechanical forces affect actin polymerization kinetics, a process that appears to be highly sensitive to the presence of profilin (Courtemanche et al., 2013). Furthermore, the assembly of branched actin networks is sensitive to mechanical load resulting in the formation of stiffer and mechanically more resistant structures under force (Bieling et al., 2016).

Given this inherent mechanosensitivity of the actin cytoskeleton, it is not surprising that also microtubule networks are susceptible to mechanical signals. Cell culture studies showed that externally applied forces can modify microtubule growth direction (Brangwynne et al., 2007). In vitro studies demonstrated that the time microtubules spend in the elongation phase during polymerization is strongly decreased when the filament polymerizes against a micro-fabricated barrier (Janson et al., 2003). Together, these data indicate that cytoskeletal networks actively integrate mechanical information.

\section{Integrating mechanical signals from distinct mechanosensitive structures}

The examples above imply that distinct mechanosensitive structures respond to mechanical stimuli in a highly specific and spatiotemporally controlled fashion. However, orchestrating a mechanoresponse in cells or even whole tissues must require the coordination of these ostensibly independent signals that often occur on different time and length scales. The application of an external stimulus may lead to the activation of MS ion channels within milliseconds (Coste et al., 2010), and - at the same time - modulate the rate of protein turnover in FAs that is typically in the order of tens of seconds (Stutchbury et al., 2017). The same mechanical stimulus may induce changes in nuclear architecture leading to altered gene transcription and protein expression over the course of several hours (Le et al., 2016). How can these different mechanoresponses be coordinated? The answer to this question is certainly complex and not easily captured by any single of the currently available cell mechanics models (Lange and Fabry, 2013; Ingber et al., 2014). However, some mechanisms appear to be especially prominent in a range of cell types including the physical interconnection of mechanosensitive elements, the use of common signaling or adaptor molecules, and the convergence of mechanochemical signal transduction cascades (Figure 2).

\section{Mechanical crosstalk through physical interconnection}

An intact cytoskeleton is a prerequisite for many of the observed cellular mechanoresponses but also serves as a physical connection between locally separated mechanosensitive elements (Figure 2A, Matthews et al., 2006). For example, regulation of cell surface tension, which modulates the activity of MS ion channels, depends on the interaction with the underlying cortical actin network (Chugh et al., 2017). Similarly, application of mechanical forces on integrin receptors or actin stress fibers results in ion fluxes (Matthews et al., 2006; Hayakawa et al., 2008) suggesting a crosstalk between cell adhesion receptors and MS ion channels. Another example of mechanical crosstalk through physical interconnection is nuclear-cytoskeletal coupling that relies on LINC proteins connecting the nucleoskeleton to actin networks, microtubules and intermediate filaments. The resulting linkages allow an almost instant transduction of mechanical information from the extracellular space to the nucleus (Lombardi et al., 2011) leading to changes in gene transcription and altered protein expression levels.

The coupling of plasma membrane tension with actin dynamics has been observed in neutrophils, where a local increase in plasma membrane tension causes a long-range inhibition of actin assembly that leads to a confined leading-edge formation (Houk et al., 2012). A membrane-actin crosstalk has been also observed in keratocytes where a rapid increase in membrane tension through micropipette aspiration in trailing regions of migrating cells leads to an increase in actin density at lamellipodial regions at the opposite side of the cell (Mueller et al., 2017). Collectively, these data show that mechanical information is efficiently 


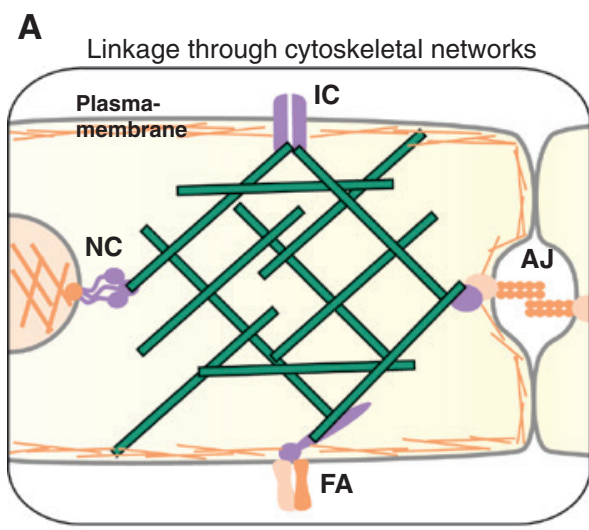

C

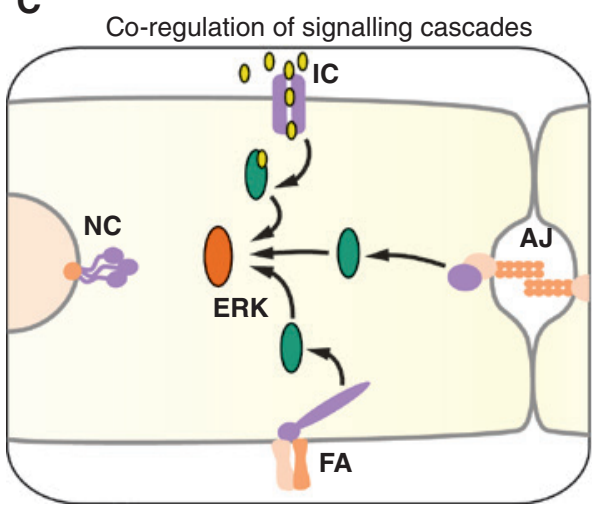

B

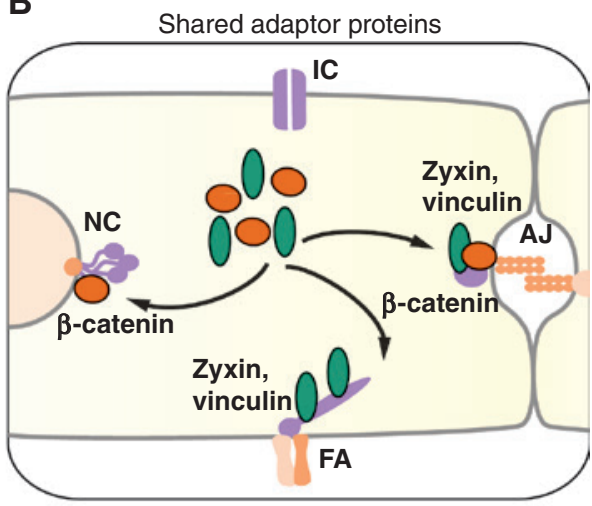

D Feedback through transcriptional regulation

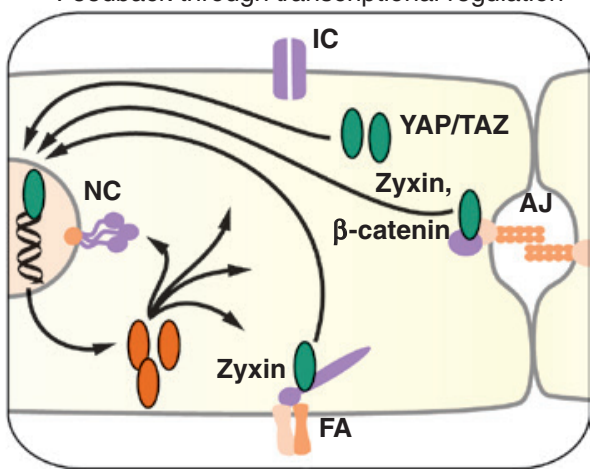

Figure 2: Mechanisms of mechanical crosstalk in mammalian cells.

(A) Linkage through cytoskeletal networks. The connection of distinct mechanosensitive structures through the plasma membrane, the cortical actin and the internal cytoskeleton allows crosstalk through physical linkage. Different mechanosensitive structures interact through interfering signaling cascades mediated by (B) shared adaptor proteins or (C) co-regulated signaling cascades. (D) Nuclear translocation of transcriptional regulators upon a mechanical stimulus may cause changes in protein expression levels that feed back on central elements of mechanosensitive complexes.

exchanged between spatially separated mechanosensitive structures.

\section{Crosstalk through common adaptor proteins and mechano-chemical signaling cascades}

Similar to classical growth factor receptors, many mechanosensitive entities are able to induce complex signaling cascades. The interaction through chemical signaling pathways constitutes an additional mode of mechanical crosstalk in cells. FAs and AJs, for instance, both connect to the actin cytoskeleton and recruit common molecules such as vinculin or zyxin (Figure 2B, Sperry et al., 2010; Bays et al., 2014) and, at least in endothelial cells, FAK (Chen et al., 2012). Zyxin has been also detected in the nucleus, where it acts as a transcriptional regulator (Nix and Beckerle, 1997). As a result, FAs and AJs talk to and are regulated by a common set of signaling cascades and transcriptional networks ensuring an efficient intracellular communication (Mui et al., 2016). The interconnection of AJs and the nuclear compartment through commonly shared proteins was demonstrated for $\beta$-catenin, which localizes as a transcriptional regulator to the nucleus and as an adaptor protein to AJs (Bienz, 2005; Gayrard et al., 2018). Intriguingly, $\beta$-catenin, is also recruited to the LINC complex upon application of mechanical stretch (Uzer et al., 2018).

Crosstalk through mechano-chemical signaling is evident for MS ion channels and cell adhesion complexes. Stretching epithelial cells leads to Piezo-dependent activation of the ERK signaling pathway, which is also modulated by integrin receptors albeit on longer time scales (Figure 2C, Gudipaty et al., 2017). Studies in breast cancer cells revealed MS ion channel-dependent activation of Akt signaling that leads to E-cadherin downregulation (Lee et al., 2017), and Piezo1 mediated $\mathrm{Ca}^{2+}$ influx was shown to regulate the proteolytic enzyme calpain, which plays an important role in the turnover of talin (Li et al., 2014).

An intriguing combination of crosstalk through cytoskeletal coupling and mechano-chemical signaling has been described for the YAP/TAZ cascade (Figure 2D). YAP (Yes-associated protein) and TAZ (transcriptional 
co-activator of PDZ-binding motif) are transcriptional regulators that shuttle between the cytoplasm and the nucleus (Halder et al., 2012). The nuclear localization and thus the strength of YAP/TAZ signaling depends on ECM rigidity and actin stress fiber formation (Dupont et al., 2011). Recent experiments revealed that the ECM stiffness-dependent regulation of YAP/TAZ localization is also sensitive to the density and spacing of integrin receptors (Oria et al., 2017), while an independent study identified a role of YAP/TAZ in regulating the stability of AJs through modulation of BMP signaling (Neto et al., 2018). It has been also speculated that nuclear entry of YAP is modulated by force-dependent regulation of nuclear pores (Elosegui-Artola et al., 2017). Thus, YAP and TAZ are versatile integrators of nuclearcytoskeletal crosstalk and mechano-chemical signaling pathways. Together, these examples show that cells adjust their mechanoresponses through interconnecting distinct force-sensitive elements and signaling cascades.

\section{The mechanical memory of cells}

A fascinating property of our tissues is their ability to remember past mechanical environments and stimulations. This mechanical memory is obviously important for tissue homeostasis, such as muscle growth after mechanical exercise, but is also observed in individual cells. Muscle stem cells, for instance, will engraft efficiently into muscle tissue only when previously cultured on substrates matching the physiological tissue stiffness (Gilbert et al., 2010). Similarly, prolonged culture of human mesenchymal stem cells on rigid substrates leads to the expected nuclear localization of YAP, but prevents YAP re-localization into the cytosol for up to 10 days even when cells are subsequently cultured in soft environments (Yang et al., 2014). Where does this mechanical memory originate?

\section{Mechanical short-term memory at the plasma membrane}

As discussed already, MS ion channels are efficient mediators of mechanical signals at the plasma membrane. In contrast to other mechanosensitive molecules, however, force-gated ion channels become insensitive to repetitive mechanical stimulations through a process called desensitization (Honore et al., 2006). How long mechanosensitive ion channels remain in the non-conducting state depends on the respective ion channel, the mechanical stimulus, and whether desensitization occurs through adaptation or inactivation: adaptation refers to the uncoupling of the channel from the mechanical stimulus, whereas inactivation refers to the block of the permeating pore. Desensitization of the hair cell resident ion channel complex, for example, is achieved through two complementary adaptation mechanisms involving $\mathrm{Ca}^{2+}$-binding to the channel complex and uncoupling of the channel associated motor myosin-1c (Fettiplace and Ricci, 2003). The MS potassium channels TREK-1 and TRAAK desensitize through an inactive state (Honore et al., 2006), and also Piezos are inactivating channels that cannot be repeatedly stimulated by mechanical forces before returning to their initial state (Coste et al., 2010). Thus, force-gated ion channels bestow cells with a shortterm memory, lasting tens to hundreds of milliseconds, which probably helps cells to distinguish differences in frequency and amplitude of a mechanical signal.

\section{Mechanical memory through cytoskeletal reorganization and conformational change}

A more enduring mechanical memory is established through the reorganization of cytoskeletal structures. On the one hand, cells exposed to external forces will stiffen their cytoskeletal networks through passive strainstiffening (Gardel et al., 2004) and active reinforcement (Matthews et al., 2006); in the case of repeated stretching, they also reorient their actin filaments to minimize future mechanical loads (Matthews et al., 2006; Faust et al., 2011). On the other hand, cells fluidize and re-solidify in response to mechanical stretch (Trepat et al., 2007). Both types of response lead to a cytoskeletal restructuring that, depending on the cell type and stimulus, may persist for minutes to hours.

Another strengthening mechanism that involves cytoskeletal reorganization and conformational memory is observed in FAs, which typically enlarge in response to applied forces (Riveline et al., 2001). This somewhat counterintuitive behavior is partly explained by the presence of special protein-protein interactions called catch bonds, which - in contrast to slip bonds - are characterized by an increased lifetime under tension. The $\alpha 5 \beta 1$ integrin receptor forms such a catch bond with its extracellular ligand fibronectin upon exposure to external stress (Kong et al., 2009). Intriguingly, repeated application of mechanical forces to the $\alpha 5 \beta 1$-FN linkage induces the switch from a short-lived catch bond, with characteristic lifetimes in the order of $1 \mathrm{~s}$, to a long-lived state with bond lifetimes of up to $100 \mathrm{~s}$ (Kong et al., 2013). In addition, mechanical information is stored within FA proteins that adjust their conformation in response to mechanical loads. The force-induced vinculin recruitment to talin, for instance, does not only 
result in the formation of force-bearing linkages to the actin network (Atherton et al., 2015; Austen et al., 2015), it also prevents talin refolding when forces decrease again (Yao et al., 2014b). As a result, FAs will respond differently to future force applications. This sort of mechanical memory through cytoskeletal reorganization and strengthening mechanisms affects cells in the order of minutes to hours.

\section{Storing mechanical information by transcriptional regulation and chromatin remodeling}

The long-term memory of cells is largely mediated by changes in gene expression through the nuclear localization of transcription factors and chromatin remodeling. The already mentioned example of persistent YAP localization in the nuclear compartment falls into this category (Yang et al., 2014). However, it remains to be investigated how retention of nuclear YAP on soft substrates is controlled. Other transcriptional regulators that alter gene expression in a force-sensitive fashion include $\beta$-catenin, which activates the transcription factor twist in epithelial cells (Farge, 2003). Serum response factor (SRF) and its co-regulator MAL govern the expression of many adhesion proteins including talin, vinculin or zyxin (Schratt et al., 2002), and NF- $\kappa B$ translocates to the nucleus of endothelial cells and osteoblasts in response to shear flow (Khachigian et al., 1995; Chen et al., 2003).

While the force-dependent localization of these transcriptional regulators usually leads to an increase in transcriptional activity, the opposite - transcriptional repression in response to mechanical strain - has also been described. In skin cells, application of cyclic stretch causes global chromatin rearrangements leading to histone 3 trimethylation at lysine 27 and dampened gene transcription (Le et al., 2016). Regardless of whether the transcriptional activity is enhanced or repressed, this type of regulation endows cells with a long-term mechanical memory that may persist for many hours or even days. Finally, physical cues modulate the differentiation of stem cells and thereby determine tissue function for years (Engler et al., 2006; Trappmann et al., 2012; Li et al., 2013).

\section{Conclusions and future perspective}

Our examples demonstrate that cells use complex mechanisms to sense, translate and respond to a wide range of distinct mechanical stimuli. In addition, cells seem to possess a sort of mechanical memory allowing them to adjust to future mechanical challenges. While tremendous progress in understanding cellular mechanotransduction has been made, it is also clear that many details of the underlying molecular mechanisms remain to be identified. How do the various MS ion channels affect each other when expressed in the same cell? How do other types of cell adhesion complexes such as tight junctions, desmosomes or hemidesmosomes process mechanical information, and what are relevant length and time scales that characterize the underlying molecular processes? The development of highly sensitive technologies such as single-molecule force spectroscopy (Rognoni et al., 2014; Grison et al., 2017), atomic force microscopy (Kong et al., 2009; Kong et al., 2013; Strohmeyer et al., 2017), and molecular tension sensors (Austen et al., 2015; Ringer et al., 2017a) will certainly be helpful to develop a quantitative understanding of molecular force transduction in cells. It will be crucial, however, to improve the applicability of these techniques to investigate processes of molecular force transduction in complex but physiologically more relevant settings, specifically mammalian tissues. Furthermore, recent developments in super-resolution microscopy hold the promise to study the molecular underpinnings of cellular mechanotransduction on the level of individual molecules in living cells and organisms (Balzarotti et al., 2017; Schueder et al., 2017). Understanding these mechanisms will not only shed light onto one of the most fundamental and evolutionary conserved properties of life, it should also allow generations of future scientists to develop cells and tissues with designed mechano-chemical properties.

Acknowledgments: The authors acknowledge funding by the German Research Council through the DFG-ANR program, Funder Id: 10.13039/501100001659 (GR 3399/6-1), the Collaborative Research Centre SFB863 (B9) and the Human Frontier Science Program (RGP0024).

\section{References}

Atherton, P., Stutchbury, B., Wang, D.Y., Jethwa, D., Tsang, R., Meiler-Rodriguez, E., Wang, P.B., Bate, N., Zent, R., Barsukov, I.L., et al. (2015). Vinculin controls talin engagement with the actomyosin machinery. Nat. Commun. 6, 10038.

Austen, K., Ringer, P., Mehlich, A., Chrostek-Grashoff, A., Kluger, C., Klingner, C., Sabass, B., Zent, R., Rief, M., and Grashoff, C. (2015). Extracellular rigidity sensing by talin isoform-specific mechanical linkages. Nat. Cell Biol. 17, 1597-1606.

Balzarotti, F., Eilers, Y., Gwosch, K.C., Gynna, A.H., Westphal, V., Stefani, F.D., Elf, J., and Hell, S.W. (2017). Nanometer resolution 
imaging and tracking of fluorescent molecules with minimal photon fluxes. Science 355, 606-612.

Bartolak-Suki, E., Imsirovic, J., Parameswaran, H., Wellman, T.J., Martinez, N., Allen, P.G., Frey, U., and Suki, B. (2015). Fluctuation-driven mechanotransduction regulates mitochondrialnetwork structure and function. Nat. Mater. 14, 1049-1057.

Baumann, F., Bauer, M.S., Rees, M., Alexandrovich, A., Gautel, M., Pippig, D.A., and Gaub, H.E. (2017). Increasing evidence of mechanical force as a functional regulator in smooth muscle myosin light chain kinase. Elife 6, pii:e26473.

Bays, J.L., Peng, X., Tolbert, C.E., Guilluy, C., Angell, A.E., Pan, Y., Superfine, R., Burridge, K., and DeMali, K.A. (2014). Vinculin phosphorylation differentially regulates mechanotransduction at cell-cell and cell-matrix adhesions. J. Cell Biol. 205, 251-263.

Berridge, M.J., Bootman, M.D., and Roderick, H.L. (2003). Calcium signalling: dynamics, homeostasis and remodelling. Nat. Rev. Mol. Cell Biol. 4, 517-529.

Bieling, P., Li, T.D., Weichsel, J., McGorty, R., Jreij, P., Huang, B., Fletcher, D.A., and Mullins, R.D. (2016). Force feedback controls motor activity and mechanical properties of self-assembling branched actin networks. Cell 164, 115-127.

Bienz, M. (2005). beta-Catenin: a pivot between cell adhesion and Wnt signalling. Curr. Biol. 15, R64-R67.

Brangwynne, C.P., MacKintosh, F.C., and Weitz, D.A. (2007). Force fluctuations and polymerization dynamics of intracellular microtubules. Proc. Natl. Acad. Sci. USA 104, 16128-16133.

Brohawn, S.G. (2015). How ion channels sense mechanical force: insights from mechanosensitive K2P channels TRAAK, TREK1, and TREK2. Ann. Reports 1352, 20-32.

Brohawn, S.G., Campbell, E.B., and MacKinnon, R. (2014a). Physical mechanism for gating and mechanosensitivity of the human TRAAK K+ channel. Nature 516, 126-130.

Brohawn, S.G., Su, Z.W., and MacKinnon, R. (2014b). Mechanosensitivity is mediated directly by the lipid membrane in TRAAK and TREK1 K+ channels. Proc. Natl. Acad. Sci. USA 111, 3614-3619.

Buckley, C.D., Tan, J.Y., Anderson, K.L., Hanein, D., Volkmann, N., Weis, W.I., Nelson, W.J., and Dunn, A.R. (2014). The minimal cadherin-catenin complex binds to actin filaments under force. Science 346, 1254211.

Chalfie, M. (2009). Neurosensory mechanotransduction. Nat. Rev. Mol. Cell Biol. 10, 44-52.

Chen, N.X., Geist, D.J., Genetos, D.C., Pavalko, F.M., and Duncan, R.L. (2003). Fluid shear-induced NF kappa B translocation in osteoblasts is mediated by intracellular calcium release. Bone 33, 399-410.

Chen, X.L., Nam, J.O., Jean, C., Lawson, C., Walsh, C.T., Goka, E., Lim, S.T., Tomar, A., Tancioni, I., Uryu, S., et al. (2012). VEGFinduced vascular permeability is mediated by FAK. Dev. Cell. 22, 146-157.

Chubinskiy-Nadezhdin, V.I., Negulyaev, Y.A., and Morachevskaya, E.A. (2011). Cholesterol depletion-induced inhibition of stretchactivated channels is mediated via actin rearrangement. Biochem. Bioph. Res. Co. 412, 80-85.

Chugh, P., Clark, A.G., Smith, M.B., Cassani, D.A.D., Dierkes, K., Ragab, A., Roux, P.P., Charras, G., Salbreux, G., and Paluch, E.K. (2017). Actin cortex architecture regulates cell surface tension. Nat. Cell Biol. 19, 689-697.

Coste, B., Mathur, J., Schmidt, M., Earley, T.J., Ranade, S., Petrus, M.J., Dubin, A.E., and Patapoutian, A. (2010). Piezo1 and Piezo2 are essential components of distinct mechanically activated cation channels. Science 330, 55-60.

Courtemanche, N., Lee, J.Y., Pollard, T.D., and Greene, E.C. (2013). Tension modulates actin filament polymerization mediated by formin and profilin. Proc. Natl. Acad. Sci. USA 110, 9752-9757.

Cueva, J.G., Mulholland, A., and Goodman, M.B. (2007). Nanoscale organization of the MEC-4 DEG/ENaC sensory mechanotransduction channel in Caenorhabditis elegans touch receptor neurons. J. Neurosci. 27, 14089-14098.

del Rio, A., Perez-Jimenez, R., Liu, R., Roca-Cusachs, P., Fernandez, J.M., and Sheetz, M.P. (2009). Stretching single talin rod molecules activates vinculin binding. Science 323, 638-641.

Doyle, A., Marganski, W., and Lee, J. (2004). Calcium transients induce spatially coordinated increases in traction force during the movement of fish keratocytes. J. Cell Sci. 117, 2203-2214.

Dupont, S., Morsut, L., Aragona, M., Enzo, E., Giulitti, S., Cordenonsi, M., Zanconato, F., Le Digabel, J., Forcato, M., Bicciato, S., et al. (2011). Role of YAP/TAZ in mechanotransduction. Nature 474, 179-183.

Elosegui-Artola, A., Andreu, I., Beedle, A.E.M., Lezamiz, A., Uroz, M., Kosmalska, A.J., Oria, R., Kechagia, J.Z., Rico-Lastres, P., Le Roux, A.L., et al. (2017). Force triggers YAP nuclear entry by regulating transport across nuclear pores. Cell 171, 1397-1410.

Engler, A.J., Sen, S., Sweeney, H.L., and Discher, D.E. (2006). Matrix elasticity directs stem cell lineage specification. Cell 126, 677-689.

Farge, E. (2003). Mechanical induction of twist in the Drosophila foregut/stomodeal primordium. Curr. Biol. 13, 1365-1377.

Faust, U., Hampe, N., Rubner, W., Kirchgessner, N., Safran, S., Hoffmann, B., and Merkel, R. (2011). Cyclic stress at $\mathrm{mHz}$ frequencies aligns fibroblasts in direction of zero strain. PLoS One 6, e28963.

Fettiplace, R. and Ricci, A.J. (2003). Adaptation in auditory hair cells. Curr. Opin. Neurobiol. 13, 446-451.

Forth, S. and Kapoor, T.M. (2017). The mechanics of microtubule networks in cell division. J. Cell Biol. 216, 1525-1531.

Galkin, V.E., Orlova, A., and Egelman, E.H. (2012). Actin filaments as tension sensors. Curr. Biol. 22, R96-R101.

Gardel, M.L., Shin, J.H., MacKintosh, F.C., Mahadevan, L., Matsudaira, P., and Weitz, D.A. (2004). Elastic behavior of crosslinked and bundled actin networks. Science 304, 1301-1305.

Gardel, M.L., Kasza, K.E., Brangwynne, C.P., Liu, J.Y., and Weitz, D.A. (2008). Mechanical response of cytoskeletal networks. Method. Cell Biol. 89, 487-519.

Gauthier, N.C., Masters, T.A., and Sheetz, M.P. (2012). Mechanical feedback between membrane tension and dynamics. Trends Cell Biol. 22, 527-535.

Gayrard, C., Bernaudin, C., Dejardin, T., Seiler, C., and Borghi, N. (2018). Src- and confinement-dependent FAK activation causes E-cadherin relaxation and beta-catenin activity. J. Cell Biol. 217, 1063-1077.

Geiger, B., Spatz, J.P., and Bershadsky, A.D. (2009). Environmental sensing through focal adhesions. Nat. Rev. Mol. Cell Biol. 10, 21-33.

Gilbert, P.M., Havenstrite, K.L., Magnusson, K.E., Sacco, A., Leonardi, N.A., Kraft, P., Nguyen, N.K., Thrun, S., Lutolf, M.P., and Blau, H.M. (2010). Substrate elasticity regulates skeletal muscle stem cell self-renewal in culture. Science 329, 1078-1081. 
Goodman, M.B., Ernstrom, G.G., Chelur, D.S., O’Hagan, R., Yao, C.A., and Chalfie, M. (2002). MEC-2 regulates C. elegans DEG/ $\mathrm{ENaC}$ channels needed for mechanosensation. Nature 417, 880.

Grison, M., Merkel, U., Kostan, J., Djinovic-Carugo, K., and Rief, M. (2017). $\alpha$-Actinin/titin interaction: A dynamic and mechanically stable cluster of bonds in the muscle Z-disk. Proc. Natl. Acad. Sci. USA 114, 1015-1020.

Gudipaty, S.A., Lindblom, J., Loftus, P.D., Redd, M.J., Edes, K., Davey, C.F., Krishnegowda, V., and Rosenblatt, J. (2017). Mechanical stretch triggers rapid epithelial cell division through Piezo1. Nature 543, 118-121.

Guet, D., Mandal, K., Pinot, M., Hoffmann, J., Abidine, Y., Sigaut, W., Bardin, S., Schauer, K., Goud, B., and Manneville, J.B. (2014). Mechanical role of actin dynamics in the rheology of the Golgi complex and in Golgi-associated trafficking events. Curr. Biol. 24, 1700-1711.

Halder, G., Dupont, S., and Piccolo, S. (2012). Transduction of mechanical and cytoskeletal cues by YAP and TAZ. Nat. Rev. Mol. Cell Biol. 13, 591-600.

Han, M.K.L. and de Rooij, J. (2016). Converging and unique mechanisms of mechanotransduction at adhesion sites. Trends Cell Biol. 26, 612-623.

Harris, A.R., Jreij, P., and Fletcher, D.A. (2018). Mechanotransduction by the actin cytoskeleton: converting mechanical stimuli into biochemical signals. Annu. Rev. Biophys. 47, 617-631.

Hayakawa, K., Tatsumi, H., and Sokabe, M. (2008). Actin stress fibers transmit and focus force to activate mechanosensitive channels. J. Cell Sci. 121, 496-503.

Hayakawa, K., Tatsumi, H., and Sokabe, M. (2011). Actin filaments function as a tension sensor by tension-dependent binding of cofilin to the filament. J. Cell Biol. 195, 721-727.

Hill, A., Zheng, X.G., Li, X.L., McKinney, R., Dickman, D., and BenShahar, Y. (2017). The Drosophila postsynaptic DEG/ENaC channel ppk29 contributes to excitatory neurotransmission. J. Neurosci. 37, 3171-3180.

Hoffman, B.D., Grashoff, C., and Schwartz, M.A. (2011). Dynamic molecular processes mediate cellular mechanotransduction. Nature 475, 316-323.

Honore, E. (2007). The neuronal background K2P channels: focus on TREK1. Nat. Rev. Neurosci. 8, 251-261.

Honore, E., Patel, A.J., Chemin, J., Suchyna, T., and Sachs, F. (2006). Desensitization of mechano-gated K2P channels. Proc. Natl. Acad. Sci. USA 103, 6859-6864.

Houk, A.R., Jilkine, A., Mejean, C.O., Boltyanskiy, R., Dufresne, E.R., Angenent, S.B., Altschuler, S.J., Wu, L.F., and Weiner, O.D. (2012). Membrane tension maintains cell polarity by confining signals to the leading edge during neutrophil migration. Cell $148,175-188$.

Huang, D.L., Bax, N.A., Buckley, C.D., Weis, W.I., and Dunn, A.R. (2017). Vinculin forms a directionally asymmetric catch bond with F-actin. Science 357, 703-706.

Humphrey, J.D., Dufresne, E.R., and Schwartz, M.A. (2014). Mechanotransduction and extracellular matrix homeostasis. Nat. Rev. Mol. Cell Biol. 15, 802-812.

Ingber, D.E., Wang, N., and Stamenovic, D. (2014). Tensegrity, cellular biophysics, and the mechanics of living systems. Rep. Prog. Phys. 77, 046603.

Jaalouk, D.E. and Lammerding, J. (2009). Mechanotransduction gone awry. Nat. Rev. Mol. Cell Biol. 10, 63-73.
Jahed, Z., Soheilypour, M., Peyro, M., and Mofrad, M.R.K. (2016). The LINC and NPC relationship - it's complicated! J. Cell Sci. 129, 3219-3229.

Janson, M.E., de Dood, M.E., and Dogterom, M. (2003). Dynamic instability of microtubules is regulated by force. J. Cell Biol. 161, 1029-1034.

Kazmierczak, P., Sakaguchi, H., Tokita, J., Wilson-Kubalek, E.M., Milligan, R.A., Muller, U., and Kachar, B. (2007). Cadherin 23 and protocadherin 15 interact to form tip-link filaments in sensory hair cells. Nature 449, 87-91.

Khachigian, L.M., Resnick, N., Gimbrone, M.A., and Collins, T. (1995). Nuclear factor-kappa-B interacts functionally with the platelet-derived growth-factor B-chain shear-stress response element in vascular endothelial-cells exposed to fluid shearstress. J. Clin. Invest. 96, 1169-1175.

Kirby, T.J. and Lammerding, J. (2018). Emerging views of the nucleus as a cellular mechanosensor. Nat. Cell Biol. 20, 373-381.

Kong, F., Garcia, A.J., Mould, A.P., Humphries, M.J., and Zhu, C. (2009). Demonstration of catch bonds between an integrin and its ligand. J. Cell Biol. 185, 1275-1284.

Kong, F., Li, Z.H., Parks, W.M., Dumbauld, D.W., Garcia, A.J., Mould, A.P., Humphries, M.J., and Zhu, C. (2013). Cyclic mechanical reinforcement of integrin-ligand interactions. Mol. Cell 49, 1060-1068.

Kuo, J.C., Han, X.M., Hsiao, C.T., Yates, J.R., and Waterman, C.M. (2011). Analysis of the myosin-II-responsive focal adhesion proteome reveals a role for beta-Pix in negative regulation of focal adhesion maturation. Nat. Cell Biol. 13, 383-93.

Laakso, J.M., Lewis, J.H., Shuman, H., and Ostap, E.M. (2008). Myosin I can act as a molecular force sensor. Science 321, 133-136.

Lange, J.R. and Fabry, B. (2013). Cell and tissue mechanics in cell migration. Exp. Cell Res. 319, 2418-2423.

Le, H.Q., Ghatak, S., Yeung, C.Y., Tellkamp, F., Gunschmann, C., Dieterich, C., Yeroslaviz, A., Habermann, B., Pombo, A., Niessen, C.M., and Wickstrom, S.A. (2016). Mechanical regulation of transcription controls polycomb-mediated gene silencing during lineage commitment. Nat. Cell Biol. 18, 864-875.

Lee, W.H., Choong, L.Y., Jin, T.H., Mon, N.N., Chong, S., Liew, C.S., Putti, T., Lu, S.Y., Harteneck, C., and Lim, Y.P. (2017). TRPV4 plays a role in breast cancer cell migration via $\mathrm{Ca}(2+)$-dependent activation of AKT and downregulation of E-cadherin cell cortex protein. Oncogenesis 6, e338.

Li, B.J., Moshfegh, C., Lin, Z., Albuschies, J., and Vogel, V. (2013). Mesenchymal stem cells exploit extracellular matrix as mechanotransducer. Sci. Rep. 3, 2425.

Li, J., Hou, B., Tumova, S., Muraki, K., Bruns, A., Ludlow, M.J., Sedo, A., Hyman, A.J., McKeown, L., Young, R.S., et al. (2014). Piezo1 integration of vascular architecture with physiological force. Nature 515, 279-282.

Lombardi, M.L., Jaalouk, D.E., Shanahan, C.M., Burke, B., Roux, K.J., and Lammerding, J. (2011). The interaction between nesprins and sun proteins at the nuclear envelope is critical for force transmission between the nucleus and cytoskeleton. J. Biol. Chem. 286, 26743-26753.

Maingret, F., Fosset, M., Lesage, F., Lazdunski, M., and Honore, E. (1999a). TRAAK is a mammalian neuronal mechano-gated K+ channel. J. Biol. Chem. 274, 1381-1387.

Maingret, F., Patel, A.J., Lesage, F., Lazdunski, M., and Honore, E. (1999b). Mechano- or acid stimulation, two interactive modes of activation of the TREK-1 potassium channel. J. Biol. Chem. 274, 26691-26696. 
Maresca, T.J. and Salmon, E.D. (2010). Welcome to a new kind of tension: translating kinetochore mechanics into a wait-anaphase signal. J. Cell Sci. 123, 825-835.

Martinac, B. (2011). Bacterial mechanosensitive channels as a paradigm for mechanosensory transduction. Cell Physiol. Biochem. 28, 1051-1060.

Martinac, B., Adler, J., and Kung, C. (1990). Mechanosensitive ion channels of Escherichia coli activated by amphipaths. Nature 348, 261-263.

Matthews, B.D., Overby, D.R., Mannix, R., and Ingber, D.E. (2006). Cellular adaptation to mechanical stress: role of integrins, Rho, cytoskeletal tension and mechanosensitive ion channels. J. Cell Sci. 119, 508-518.

Mazzochi, C., Bubien, J.K., Smith, P.R., and Benos, D.J. (2006). The carboxyl terminus of the alpha-subunit of the amiloride-sensitive epithelial sodium channel binds to F-actin. J. Biol. Chem. $281,6528-6538$.

Moroni, M., Servin-Vences, M.R., Fleischer, R., Sanchez-Carranza, O., and Lewin, G.R. (2018). Voltage gating of mechanosensitive PIEZO channels. Nat. Commun. 9, 1096.

Mueller, J., Szep, G., Nemethova, M., de Vries, I., Lieber, A.D., Winkler, C., Kruse, K., Small, J.V., Schmeiser, C., Keren, K., et al. (2017). Load adaptation of lamellipodial actin networks. Cell 171, 188-200.

Mui, K.L., Chen, C.S., and Assoian, R.K. (2016). The mechanical regulation of integrin-cadherin crosstalk organizes cells, signaling and forces. J. Cell Sci. 129, 1093-1100.

Neto, F., Klaus-Bergmann, A., Ong, Y.T., Alt, S., Vion, A.C., Szymborska, A., Carvalho, J.R., Hollfinger, I., Bartels-Klein, E., Franco, C.A., et al. (2018). YAP and TAZ regulate adherens junction dynamics and endothelial cell distribution during vascular development. Elife 7, pii: e31037.

Nix, D.A. and Beckerle, M.C. (1997). Nuclear-cytoplasmic shuttling of the focal contact protein, zyxin: A potential mechanism for communication between sites of cell adhesion and the nucleus. J. Cell Biol. 138, 1139-1147.

Nonomura, K., Woo, S.H., Chang, R.B., Gillich, A., Qiu, Z., Francisco, A.G., Ranade, S.S., Liberles, S.D., and Patapoutian, A. (2017). Piezo2 senses airway stretch and mediates lung inflationinduced apnoea. Nature 541, 176-181.

Oria, R., Wiegand, T., Escribano, J., Elosegui-Artola, A., Uriarte, J.J., Moreno-Pulido, C., Platzman, I., Delcanale, P., Albertazzi, L., Navajas, D., et al. (2017). Force loading explains spatial sensing of ligands by cells. Nature 552, 219-224.

Pasapera, A.M., Schneider, I.C., Rericha, E., Schlaepfer, D.D., and Waterman, C.M. (2010). Myosin II activity regulates vinculin recruitment to focal adhesions through FAK-mediated paxillin phosphorylation. J. Cell Biol. 188, 877-890.

Pelham, R.J., Jr. and Wang, Y. (1997). Cell locomotion and focal adhesions are regulated by substrate flexibility. Proc. Natl. Acad. Sci. USA 94, 13661-13665.

Perozo, E., Cortes, D.M., Sompornpisut, P., Kloda, A., and Martinac, B. (2002). Open channel structure of MscL and the gating mechanism of mechanosensitive channels. Nature 418, 942-948.

Poh, Y.C., Shevtsov, S.P., Chowdhury, F., Wu, D.C., Na, S., Dundr, M., and Wang, N. (2012). Dynamic force-induced direct dissociation of protein complexes in a nuclear body in living cells. Nat. Commun. 3, 866.

Qi, Y.M., Andolfi, L., Frattini, F., Mayer, F., Lazzarino, M., and Hu, J. (2015). Membrane stiffening by STOML3 facilitates mechanosensation in sensory neurons. Nat. Commun. 6, 8512.
Ranade, S.S., Syeda, R., and Patapoutian, A. (2015). Mechanically activated ion channels. Neuron 87, 1162-1179.

Ranade, S.S., Qiu, Z.Z., Woo, S.H., Hur, S.S., Murthy, S.E., Cahalan, S.M., Xu, J., Mathur, J., Bandell, M., Coste, B., et al. (2014a). Piezo1, a mechanically activated ion channel, is required for vascular development in mice. Proc. Natl. Acad. Sci. USA 111, 10347-10352.

Ranade, S.S., Woo, S.H., Dubin, A.E., Moshourab, R.A., Wetzel, C., Petrus, M., Mathur, J., Begay, V., Coste, B., Mainquist, J., et al. (2014b). Piezo2 is the major transducer of mechanical forces for touch sensation in mice. Nature 516, 121-5.

Retailleau, K., Duprat, F., Arhatte, M., Ranade, S.S., Peyronnet, R., Martins, J.R., Jodar, M., Moro, C., Offermanns, S., Feng, Y.Y., et al. (2015). Piezo1 in smooth muscle cells is involved in hypertension-dependent arterial remodeling. Cell Rep. 13, 1161-1171.

Ringer, P., Colo, G., Fassler, R., and Grashoff, C. (2017a). Sensing the mechano-chemical properties of the extracellular matrix. Matrix Biol. 64, 6-16.

Ringer, P., Weissl, A., Cost, A.L., Freikamp, A., Sabass, B., Mehlich, A., Tramier, M., Rief, M., and Grashoff, C. (2017b). Multiplexing molecular tension sensors reveals piconewton force gradient across talin-1. Nat. Methods 14, 1090-1096.

Riveline, D., Zamir, E., Balaban, N.Q., Schwarz, U.S., Ishizaki, T., Narumiya, S., Kam, Z., Geiger, B., and Bershadsky, A.D. (2001). Focal contacts as mechanosensors: Externally applied local mechanical force induces growth of focal contacts by an mDia1dependent and ROCK-independent mechanism. J. Cell Biol. 153, 1175-1185.

Roberts, G.C.K. and Critchley, D.R. (2009). Structural and biophysical properties of the integrin-associated cytoskeletal protein talin. Biophys. Rev. 1, 61-69.

Rognoni, L., Most, T., Zoldak, G., and Rief, M. (2014). Force-dependent isomerization kinetics of a highly conserved proline switch modulates the mechanosensing region of filamin. Proc. Natl. Acad. Sci. USA 111, 5568-5573.

Rottner, K., Hall, A., and Small, J.V. (1999). Interplay between Rac and Rho in the control of substrate contact dynamics. Curr. Biol. 9, 640-648.

Rubsam, M., Broussard, J.A., Wickstrom, S.A., Nekrasova, O., Green, K.J., and Niessen, C.M. (2017). Adherens junctions and desmosomes coordinate mechanics and signaling to orchestrate tissue morphogenesis and function: an evolutionary perspective. Cold Spring Harb. Perspect. Biol. 10, pii: a029207.

Sawada, Y., Tamada, M., Dubin-Thaler, B.J., Cherniavskaya, O., Sakai, R., Tanaka, S., and Sheetz, M.P. (2006). Force sensing by mechanical extension of the Src family kinase substrate p130Cas. Cell 127, 1015-1026.

Schiller, H.B., Friedel, C.C., Boulegue, C., and Fassler, R. (2011). Quantitative proteomics of the integrin adhesome show a myosin II-dependent recruitment of LIM domain proteins. Embo. Rep. 12, 259-266.

Schratt, G., Philippar, U., Berger, J., Schwarz, H., Heidenreich, O., and Nordheim, A. (2002). Serum response factor is crucial for actin cytoskeletal organization and focal adhesion assembly in embryonic stem cells. J. Cell Biol. 156, 737-750.

Schueder, F., Lara-Gutierrez, J., Beliveau, B.J., Saka, S.K., Sasaki, H.M., Woehrstein, J.B., Strauss, M.T., Grabmayr, H., Yin, P., and Jungmann, R. (2017). Multiplexed 3D super-resolution imaging of whole cells using spinning disk confocal microscopy and DNA-PAINT. Nat. Commun. 8, 2090. 
Sharif-Naeini, R., Folgering, J.H., Bichet, D., Duprat, F., Lauritzen, I., Arhatte, M., Jodar, M., Dedman, A., Chatelain, F.C., Schulte, U., et al. (2009). Polycystin-1 and -2 dosage regulates pressure sensing. Cell 139, 587-596.

Siemens, J., Lillo, C., Dumont, R.A., Reynolds, A., Williams, D.S., Gillespie, P.G., and Muller, U. (2004). Cadherin 23 is a component of the tip link in hair-cell stereocilia. Nature 428, 950-955.

Sperry, R.B., Bishop, N.H., Bramwell, J.J., Brodeur, M.N., Carter, M.J., Fowler, B.T., Lewis, Z.B., Maxfield, S.D., Staley, D.M., Vellinga, R.M. et al. (2010). Zyxin controls migration in epithelial-mesenchymal transition by mediating actin-membrane linkages at cell-cell junctions. J. Cell Physiol. 222, 612-624.

Strohmeyer, N., Bharadwaj, M., Costell, M., Fassler, R., and Muller, D.J. (2017). Fibronectin-bound alpha alpha 5 beta 1 integrins sense load and signal to reinforce adhesion in less than a second. Nat. Mater. 16, 1262-1270.

Stroud, M.J., Banerjee, I., Veevers, J., and Chen, J. (2014). Linker of nucleoskeleton and cytoskeleton complex proteins in cardiac structure, function, and disease. Circ. Res. 114, 538-548.

Stutchbury, B., Atherton, P., Tsang, R., Wang, D.Y., and Ballestrem, C. (2017). Distinct focal adhesion protein modules control different aspects of mechanotransduction. J. Cell Sci. 130, 1612-1624.

Swift, J., Ivanovska, I.L., Buxboim, A., Harada, T., Dingal, P.C., Pinter, J., Pajerowski, J.D., Spinler, K.R., Shin, J.W., Tewari, M., et al. (2013). Nuclear lamin-A scales with tissue stiffness and enhances matrix-directed differentiation. Science 341 , 1240104.

Tajik, A., Zhang, Y.J., Wei, F.X., Sun, J., Jia, Q., Zhou, W.W., Singh, R., Khanna, N., Belmont, A.S., and Wang, N. (2016). Transcription upregulation via force-induced direct stretching of chromatin. Nat. Mater. 15, 1287-1296.

Trappmann, B., Gautrot, J.E., Connelly, J.T., Strange, D.G., Li, Y., Oyen, M.L., Cohen Stuart, M.A., Boehm, H., Li, B., Vogel, V., et al. (2012). Extracellular-matrix tethering regulates stem-cell fate. Nat. Mater. 11, 642-649.

Trepat, X., Deng, L.H., An, S.S., Navajas, D., Tschumperlin, D.J., Gerthoffer, W.T., Butler, J.P., and Fredberg, J.J. (2007). Universal physical responses to stretch in the living cell. Nature 447, 592-595.

Uyeda, T.Q.P., Iwadate, Y., Umeki, N., Nagasaki, A., and Yumura, S. (2011). Stretching actin filaments within cells enhances their affinity for the myosin ii motor domain. PLoS One 6, e26200.

Uzer, G., Bas, G., Sen, B., Xie, Z.H., Birks, S., Olcum, M., McGrath, C., Styner, M., and Rubin, J. (2018). Sun-mediated mechanical LINC between nucleus and cytoskeleton regulates beta catenin nuclear access. J. Biomech. 74, 32-40.

Woo, S.H., Lukacs, V., de Nooij, J.C., Zaytseva, D., Criddle, C.R., Francisco, A., Jessell, T.M., Wilkinson, K.A., and Patapoutian, A. (2015). Piezo2 is the principal mechanotransduction channel for proprioception. Nat. Neurosci. 18, 1756-1762.

Worman, H.J. (2012). Nuclear lamins and laminopathies. J. Pathol. 226, 316-325.

Yang, C., Tibbitt, M.W., Basta, L., and Anseth, K.S. (2014). Mechanical memory and dosing influence stem cell fate. Nat. Mater. 13, 645-652.

Yao, M., Goult, B.T., Klapholz, B., Hu, X., Toseland, C.P., Guo, Y., Cong, P., Sheetz, M.P., and Yan, J. (2016). The mechanical response of talin. Nat. Commun. 7, 11966.

Yao, M., Goult, B.T., Chen, H., Cong, P., Sheetz, M.P., and Yan, J. (2014a). Mechanical activation of vinculin binding to talin locks talin in an unfolded conformation. Sci. Rep. 4, 4610.

Yao, M., Qiu, W., Liu, R., Efremov, A.K., Cong, P., Seddiki, R., Payre, M., Lim, C.T., Ladoux, B., Mege, R.M., et al. (2014b). Forcedependent conformational switch of alpha-catenin controls vinculin binding. Nat. Commun. 5, 4525.

Yonemura, S., Wada, Y., Watanabe, T., Nagafuchi, A., and Shibata, M. (2010). alpha-Catenin as a tension transducer that induces adherens junction development. Nat. Cell Biol. 12, 533-542.

Zhang, W., Cheng, L.E., Kittelmann, M., Li, J.F., Petkovic, M., Cheng, T., Jin, P., Guo, Z.H., Gopfert, M.C., Jan, L.Y., et al. (2015). Ankyrin repeats convey force to gate the NOMPC mechanotransduction channel. Cell 162, 1391-1403.

Zhou, J., Aponte-Santamaria, C., Sturm, S., Bullerjahn, J.T., Bronowska, A., and Grater, F. (2015). Mechanism of focal adhesion kinase mechanosensing. PLoS Comput. Biol. 11, e1004593. 